\title{
On the Sequential Quadratically Constrained Quadratic Programming Methods
}

\author{
M. V. Solodov \\ Instituto de Matemática Pura e Aplicada, Estrada Dona Castorina 110, Jardim Botânico, \\ Rio de Janeiro, RJ 22460-320, Brazil, solodov@impa.br
}

\begin{abstract}
An iteration of the sequential quadratically constrained quadratic programming method (SQCQP) consists of minimizing a quadratic approximation of the objective function subject to quadratic approximation of the constraints, followed by a line search in the obtained direction. Methods of this class are receiving attention due to the development of efficient interior point techniques for solving subproblems with this structure, via formulating them as second-order cone programs. Recently, Fukushima et al. (2003) proposed a SQCQP method for convex minimization with twice continuously differentiable data. Their method possesses global and locally quadratic convergence, and it is free of the Maratos effect. The feasibility of subproblems in their method is enforced by switching between the linear and quadratic approximations of the constraints. This strategy requires computing a strictly feasible point, as well as choosing some further parameters. We propose a SQCQP method where feasibility of subproblems is ensured by introducing a slack variable and, hence, is automatic. In addition, we do not assume convexity of the objective function or twice differentiability of the problem data. While our method has all the desirable convergence properties, it is easier to implement. Among other things, it does not require computing a strictly feasible point, which is a nontrivial task. In addition, its global convergence requires weaker assumptions.
\end{abstract}

Key words: quadratically constrained quadratic programming; nonsmooth penalty function; Maratos effect MSC2000 subject classification: Primary: 90C30, 90C33

OR/MS subject classification: Primary: Programming/nonlinear

History: Received May 15, 2002; revised March 19, 2003.

1. Introduction. We consider the problem

$$
\begin{array}{ll}
\underset{x}{\operatorname{minimize}} & f(x), \\
\text { subject to } & g_{i}(x) \leq 0, \quad i=1, \ldots, m,
\end{array}
$$

where $f: \Re^{n} \rightarrow \Re$ and $g: \Re^{n} \rightarrow \Re^{m}$ are differentiable functions with Lipschitz continuous derivatives. We further assume that $g$ is convex and that the Slater constraint qualification (Mangasarian 1969) is satisfied:

$$
\exists \hat{x} \in \Re^{n} \quad \text { such that } g_{i}(\hat{x})<0, \quad i=1, \ldots, m .
$$

For local rate of convergence results, $f, g$ will further be assumed to be twice continuously differentiable, and $f$ will also be assumed to be convex. We emphasize that those additional assumptions will not be needed for global convergence of our algorithm.

For the case where both $f$ and $g$ are convex twice continuously differentiable functions, Fukushima et al. (2003) proposed an SQCQP method based on solving the subproblems of the following structure:

$$
\begin{array}{ll}
\underset{d}{\operatorname{minimize}} & \left\langle f^{\prime}\left(x^{k}\right), d\right\rangle+\frac{1}{2}\left\langle H^{k} d, d\right\rangle, \\
\text { subject to } & g_{i}\left(x^{k}\right)+\left\langle g_{i}^{\prime}\left(x^{k}\right), d\right\rangle+\frac{\alpha_{i}^{k}}{2}\left\langle g_{i}^{\prime \prime}\left(x^{k}\right) d, d\right\rangle \leq 0, \quad i=1, \ldots, m,
\end{array}
$$

where $x^{k} \in \Re^{n}$ is the current iterate, $H^{k}$ is an $n \times n$ symmetric positive definite matrix, and $\alpha_{i}^{k} \in[0,1]$ are parameters which control the feasibility of (3) according to the rules described below. Subproblem (3) is a convex quadratically constrained quadratic program, which can be cast as a second-order cone program (Lobo et al. 1998, Nesterov and Nemirovskii 1993). The latter can be efficiently solved by interior point algorithms (such 
as Monteiro and Tsuchiya 2000, Tsuchiya 1999), which justifies the interest in methods based on second-order approximation of the constraints. In fact, in Anitescu (2002b) nonconvex QCQPs were also handled quite efficiently by using other nonlinear programming techniques.

Let $d^{k} \in \Re^{n}$ be the solution of (3), and let $\mu^{k} \in \Re_{+}^{m}$ be some associated Lagrange multiplier. The next iterate in the method of Fukushima et al. (2003) is given by $x^{k+1}=x^{k}+\gamma_{k} d^{k}$, where the step size $\gamma_{k}>0$ is computed using an Armijo-type linesearch procedure for the standard $l_{1}$-penalty function $f(x)+\beta_{k} \sum_{i=1}^{m}\left[g_{i}(x)\right]_{+}$, with $\beta_{k}>\left\|\mu^{k}\right\|_{\infty}$ being the penalty parameter and $[\cdot]_{+}=\max \{0, \cdot\}$. In Fukushima et al. (2003), the method outlined above is shown to be globally convergent to a solution of (1) under certain natural assumptions. Local quadratic rate of convergence is established under the assumption of positive definiteness of the Hessian of the Lagrangian of (1) at the KKT points. As discussed in Fukushima et al. (2003), one attractive feature of this SQCQP method is that it is free from the Maratos effect. The Maratos effect (Maratos 1978, Powell 1983) is a notorious difficulty for sequential quadratic programming methods (SQP) (Bertsekas 1995, Boggs and Tolle 1996, Bonnans et al. 2003), which can be thought of as a special case of SQCQP with $\alpha_{i}^{k}=0$ for all $i$ and $k$. It refers to the situation where, even though conditions for local superlinear convergence of the pure SQP iterations are satisfied, the linesearch procedure based on a nonsmooth penalty function (such as $l_{1}$ ) does not accept the unit step size. As a result, superlinear convergence is not achieved. Known remedies to avoid the Maratos effect in SQP methods are quite complex and require careful implementation (Mayne and Polak 1982, Fukushima 1986, Bonnans 1989, Bonnans et al. 2003). By contrast, no special care is needed in the SQCQP method of Fukushima et al. (2003). In particular, the unit step size is always accepted under the assumptions required for the local superlinear rate of convergence.

One important detail which may not be so easy in the implementation of the method of Fukushima et al. (2003) concerns the control of parameters $\alpha_{i}^{k}$ in (3). Note that quadratic approximation of constraints can be "too optimistic," in the sense that the feasible set in (3) can be empty if $\alpha_{i}^{k}=1$ for all $i$. By contrast, the linear approximation corresponding to $\alpha_{i}^{k}=0$ for all $i$, is guaranteed to yield feasible subproblems (in the case of convex $g$ ). The following rule is proposed in Fukushima et al. (2003) to take care of the feasibility problem:

Compute $\hat{x} \in \Re^{n}$ satsifying the Slater condition (2). Choose $\theta \in[0,1)$ and $\nu \in(\theta, 1)$. (This is done once, to initialize the algorithm.)

$$
\begin{gathered}
\text { Compute } \eta_{i}^{k}:=\left\langle g_{i}^{\prime \prime}\left(x^{k}\right)\left(x^{k}-\hat{x}\right), x^{k}-\hat{x}\right\rangle, \quad i=1, \ldots, m, \quad \text { and } \\
K_{1}\left(x^{k}\right):=\left\{i \mid g_{i}\left(x^{k}\right)>0\right\}, \quad K_{2}\left(x^{k}\right):=\left\{i \mid \theta g_{i}(\hat{x}) \leq g_{i}\left(x^{k}\right)\right\}, \\
s_{1}\left(x^{k}\right):=\max _{i \in K_{1}\left(x^{k}\right)} \frac{g_{i}\left(x^{k}\right)}{g_{i}\left(x^{k}\right)-\nu g_{i}(\hat{x})} \quad\left(s_{1}\left(x^{k}\right):=-\infty \text { if } K_{1}\left(x^{k}\right)=\varnothing\right), \\
s_{2}\left(x^{k}\right):=\min \left\{1, \min _{i \in K_{2}\left(x^{k}\right)} \frac{g_{i}\left(x^{k}\right)-\nu g_{i}(\hat{x})}{\eta_{i}^{k}}\right\}, \\
s_{3}\left(x^{k}\right):=\min \left\{s_{2}\left(x^{k}\right), \min _{i \notin K_{2}\left(x^{k}\right)} \frac{-2(\nu-\theta) g_{i}(\hat{x})}{\eta_{i}^{k}}\right\} .
\end{gathered}
$$

If $s_{2}\left(x^{k}\right) \leq 2 s_{1}\left(x^{k}\right)$ then set $\alpha_{i}^{k}=0$ for all $i$. If $s_{3}\left(x^{k}\right)>2 s_{1}\left(x^{k}\right)$ then set $\alpha_{i}^{k}=1$ for all $i$.

$$
\text { If } s_{3}\left(x^{k}\right) \leq 2 s_{1}\left(x^{k}\right)<s_{2}\left(x^{k}\right) \text {, then } \alpha_{i}^{k}=1 \text { for } i \in K_{2}\left(x^{k}\right) \text { and } \alpha_{i}^{k}=0 \text { for } i \notin K_{2}\left(x^{k}\right) \text {. }
$$

The above rule for choosing $\alpha_{i}^{k}$ is based on testing constraints violation and curvature at the current iterate, and it guarantees that the subproblem (3) is feasible. Another potentially useful feature of this rule is that it allows to balance the numbers of quadratic and linear 
constraints in the subproblem at the early stages of the algorithm (subproblems with fewer quadratic constraints are in general easier to solve). But it is guaranteed that eventually, $\alpha_{i}^{k}=1$ for all $i$, so that all the second-order information is being used. As discussed in Fukushima et al. (2003), many other rules are possible, including the continuous choice in the interval $[0,1]$. Furthermore, there are some choices to be made even for the given rule, namely the parameters $\theta, \nu$ and, especially, $\hat{x}$. The implementation is therefore not straightforward, and may require a fair bit of fine-tuning. Most importantly, computing $\hat{x}$ is a general convex feasibility problem, which is a computationally nontrivial task. Essentially, the method of Fukushima et al. (2003) is a "Phase I-Phase II" method. Phase I corresponds to computing a Slater point, while Phase II is the SQCQP method for solving problem (1). Computing a Slater point can be done in a finite number of iterations by various means, see Fukushima et al. (2003). Nevertheless, this Phase I problem has complexity comparable to the original problem itself, and so it increases the computational burden considerably. As an alternative, Fukushima et al. (2003) propose to use in the rule for choosing $\alpha_{i}^{k}$ some estimates $c_{1}$ and $c_{2}$ such that $0>c_{1} \geq \max _{i} g_{i}(\hat{x}), c_{2} \geq \max _{i}\left\langle g_{i}^{\prime \prime}\left(x^{k}\right)\left(x^{k}-\hat{x}\right), x^{k}-\hat{x}\right\rangle$ for some (unknown) Slater point $\hat{x}$. However, this requires either essentially heuristic considerations or computing the estimates iteratively (if $c_{1}$ and $c_{2}$ are not adequate and the subproblems are still infeasible, one has to increase $c_{1}$ and $c_{2}$ and try again).

In this paper, we propose to deal with the feasibility of subproblems by introducing a slack variable, which simplifies the implementation considerably. Among other things, computing a strictly feasible point is no longer necessary. In addition, for the global convergence result we do not assume the convexity of $f$ or twice differentiability of $f$ and $g$. In fact, our global convergence result appears to be stronger than that in Fukushima et al. (2003) also in a number of other ways (see the comments preceding Theorem 6).

Our proposal is to consider, instead of (3), the subproblem

$$
\begin{aligned}
& \underset{(d, t)}{\operatorname{minimize}}\left\langle f^{\prime}\left(x^{k}\right), d\right\rangle+\frac{1}{2}\left\langle H^{k} d, d\right\rangle+\beta_{k} t, \\
& \text { subject to } g_{i}\left(x^{k}\right)+\left\langle g_{i}^{\prime}\left(x^{k}\right), d\right\rangle+\frac{1}{2}\left\langle G_{i}^{k} d, d\right\rangle \leq t, \quad i \in I_{k}, t \geq 0,
\end{aligned}
$$

where $\beta_{k}>0$ is a penalty parameter, $G_{i}^{k}, i \in I_{k}$, are $n \times n$ symmetric positive semidefinite matrices (possibly different from $g_{i}^{\prime \prime}\left(x^{k}\right)$ even when the latter exists), and $I_{k}$ is any index set that satisfies

$$
\begin{gathered}
I\left(x^{k}\right) \subset I_{k} \subset\{1, \ldots, m\}, \\
I\left(x^{k}\right)=\left\{i \mid g_{i}\left(x^{k}\right)=p\left(x^{k}\right)\right\}, \quad p\left(x^{k}\right)=\max _{i=1, \ldots, m}\left[g_{i}\left(x^{k}\right)\right]_{+} .
\end{gathered}
$$

Note that the possibility to choose the index set $I_{k}$ smaller than $\{1, \ldots, m\}$ is very useful (especially at the early stages of the algorithm), because it reduces the number of constraints thus leading to simpler subproblems. This effect can be considered similar to setting $\alpha_{i}^{k}=0$ for some $i$ in (3), but can have a further advantage of removing altogether the constraints which are locally irrelevant.

As suggested by a referee, instead of (4) we could also consider subproblems with a different slack and a different penalty parameter for each of the constraints:

$$
\begin{aligned}
& \underset{(d, t)}{\operatorname{minimize}}\left\langle f^{\prime}\left(x^{k}\right), d\right\rangle+\frac{1}{2}\left\langle H^{k} d, d\right\rangle+\sum_{i \in I_{k}} \beta_{k}^{i} t_{i}, \\
& \text { subject to } g_{i}\left(x^{k}\right)+\left\langle g_{i}^{\prime}\left(x^{k}\right), d\right\rangle+\frac{1}{2}\left\langle G_{i}^{k} d, d\right\rangle \leq t_{i}, \quad t_{i} \geq 0, i \in I_{k} .
\end{aligned}
$$

Numerically, this can be advantageous in some situations. For the subsequent analysis we prefer the simpler form of (4). It can be seen that the analysis extends to the case of different slacks and penalty parameters after simple modifications. 
Let $\left(d^{k}, t^{k}\right) \in \Re^{n} \times \Re$ be the solution of (4), which is easily seen to be unique (§2). Then the next iterate of our method is given by $x^{k+1}=x^{k}+\gamma_{k} d^{k}$, where the step size $\gamma_{k}>0$ is computed using an Armijo-type linesearch procedure (see Algorithm 1) for the $l_{\infty}$-penalty function

$$
\psi_{\beta_{k}}(x)=f(x)+\beta_{k} p(x) .
$$

The idea of using a slack variable to ensure feasibility comes from a similar strategy for SQP (Bertsekas 1995). In general SQP methods, removing the feasibility concerns using this strategy comes at the expense of the difficulty in choosing the penalty parameter $\beta_{k}$. (Essentially, one needs to ensure that eventually $\beta_{k}>\left\|\mu^{k}\right\|_{1}$, where $\mu^{k}$ is a Lagrange multiplier associated with $d^{k}$. But $\beta_{k}$ has to be chosen before the new multiplier $\mu^{k}$ is computed, which leads to certain difficulties. See, for example, the the last comment in $\S 3$ of Anitescu 2002a.) For our algorithm, we are able to give a novel rule for updating the penalty parameter $\beta_{k}$ (see Algorithm 1), which guarantees convergence.

To conclude this section, we cite some previous work on SQCQP and related methods. For the first time methods of this class were probably considered in Panin $(1979,1981)$, where strong convexity of $f$ and $g$ is assumed. In Anitescu (2002b), local superlinear rate of convergence of a trust-region SQCQP method is obtained without the convexity assumptions, under the Mangasarian-Fromovitz constraint qualification and a certain quadratic growth condition. This reference also provides some numerical testing, which highlights the following fact. Although SQCQP subproblems are more computationally difficult than subproblems in SQP, they can be solved reasonably efficiently even in the nonconvex case (in the nonconvex case, subproblems have to be solved by approaches other than the interior point methods). Moreover, because SQCQP subproblems are a better approximation of the original problem, fewer subproblems have to be solved. Thus this extra effort (compared to SQP) may be well worth it. However, global convergence is not discussed in Anitescu (2002b). For some other SQCQP-related work, we mention Wiest and Polak (1992) and Kruk and Wolkowicz (2000).

Some final words about our notation. For a directionally differentiable function $\psi: \Re^{n} \rightarrow \Re$, by $\psi^{\prime}(x ; d)$ we denote the usual directional derivative of $\psi$ at the point $x \in \Re^{n}$ in the direction $d \in \Re^{n}$. By $\|\cdot\|$ we denote the 2-norm, by $\|\cdot\|_{1}$ we denote the $l_{1}$-norm, and by $\|\cdot\|_{\infty}$ the $l_{\infty}$-norm. The space is always clear from the context, and will not be specified. For two symmetric positive semidefinite matrices $A$ and $B$, we write $A \succeq B$ if the matrix $A-B$ is positive semidefinite. By $E$ we denote the identity matrix and by $O$ the zero matrix. Finally, the operation $t / 0$, where $t>0$ is considered well defined, with the result being $+\infty$.

2. SQCQP method and its global convergence. First, note that (4) is always feasible and has unique solution. Indeed, as is easy to see, for each $d \in \mathfrak{R}^{n}$ fixed, the minimum with respect to $t$ in (4) is attained at

$$
t^{k}(d)=\max _{i \in I_{k}}\left[g_{i}\left(x^{k}\right)+\left\langle g_{i}^{\prime}\left(x^{k}\right), d\right\rangle+\frac{1}{2}\left\langle G_{i}^{k} d, d\right\rangle\right]_{+} .
$$

Hence, (4) is equivalent to

$$
\underset{d}{\operatorname{minimize}}\left\langle f^{\prime}\left(x^{k}\right), d\right\rangle+\frac{1}{2}\left\langle H^{k} d, d\right\rangle+\beta_{k} t^{k}(d) .
$$

Because $H^{k}$ is positive definite, the objective function of this program is strongly convex and has the unique minimizer $d^{k}$. It follows that $\left(d^{k}, t^{k}\right)$ with $t^{k}=t^{k}\left(d^{k}\right)$ is the unique solution of (4). Furthermore, because the constraints in (4) obviously satisfy the Slater constraint 
qualification, the Karush-Kuhn-Tucker (KKT) optimality conditions (Mangasarian 1969) hold: there exist some $\mu_{i}^{k} \in \Re, i \in I_{k}$, and $\nu^{k} \in \Re$ such that

$$
\begin{gathered}
f^{\prime}\left(x^{k}\right)+H^{k} d^{k}+\sum_{i \in I_{k}} \mu_{i}^{k}\left(g_{i}^{\prime}\left(x^{k}\right)+G_{i}^{k} d^{k}\right)=0, \\
\beta_{k}-\sum_{i \in I_{k}} \mu_{i}^{k}-\nu^{k}=0, \\
g_{i}\left(x^{k}\right)+\left\langle g_{i}^{\prime}\left(x^{k}\right), d^{k}\right\rangle+\frac{1}{2}\left\langle G_{i}^{k} d^{k}, d^{k}\right\rangle \leq t^{k}, \quad \mu_{i}^{k} \geq 0, \quad i \in I_{k}, \\
\mu_{i}^{k}\left(g_{i}\left(x^{k}\right)+\left\langle g_{i}^{\prime}\left(x^{k}\right), d^{k}\right\rangle+\frac{1}{2}\left\langle G_{i}^{k} d^{k}, d^{k}\right\rangle-t^{k}\right)=0, \quad i \in I_{k}, \\
t^{k} \geq 0, \quad \nu^{k} \geq 0, \quad t^{k} \nu^{k}=0 .
\end{gathered}
$$

We proceed to formally state our algorithm.

Algorithm 1 (SQCQP). Choose some $x^{0} \in \Re^{n}, \beta_{0}, \delta_{1}, \delta_{2} \in(0,+\infty)$ and $\sigma, \theta \in(0,1)$. Set $k:=0$.

1. QCQP subproblem.

Choose an index set $I_{k}$ according to (5). Choose $n \times n$ symmetric matrices $H^{k}$ (positive definite) and $G_{i}^{k}, i \in I_{k}$ (positive semidefinite). Compute $\left(d^{k}, t^{k}\right)$ as the solution of (4), and the associated Lagrange multipliers $\left(\mu^{k}, \nu^{k}\right)$.

2. Stopping test.

Stop if $d^{k}=0$ and $t^{k}=0$.

3. Linesearch.

If $d^{k}=0$ (but $t_{k}>0$ ), set $j_{k}:=0$ and go to Step 4 .

Otherwise, find $j_{k}$, the smallest nonnegative integer $j$, such that

$$
\psi_{\beta_{k}}\left(x^{k}+\theta^{j} d^{k}\right) \leq \psi_{\beta_{k}}\left(x^{k}\right)+\sigma \theta^{j} \Delta_{k},
$$

where

$$
\Delta_{k}:=\left\langle f^{\prime}\left(x^{k}\right), d^{k}\right\rangle+\frac{1}{2}\left\langle H^{k} d^{k}, d^{k}\right\rangle+\beta_{k}\left(t^{k}-p\left(x^{k}\right)\right) .
$$

4. Variable update.

Set $\gamma_{k}:=\theta^{j_{k}}$ and $x^{k+1}:=x^{k}+\gamma_{k} d^{k}$.

5. Penalty parameter update.

Compute $r_{k}:=\min \left\{\left\|d^{k}\right\|^{-1},\left\|\mu^{k}\right\|_{1}+\delta_{1}\right\}$. Set

$$
\beta_{k+1}:=\left\{\begin{array}{ll}
\beta_{k}, & \text { if } \beta_{k} \geq r_{k} \\
\beta_{k}+\delta_{2}, & \text { if } \beta_{k}<r_{k}
\end{array} .\right.
$$

6. Set $k:=k+1$ and go to Step 1 .

Note that our penalty parameter rule is somewhat different from the usual strategies in SQP (where typically $r_{k}=\left\|\mu^{k}\right\|_{1}+\delta_{1}$ is used). As already mentioned above, choosing this parameter in the framework where feasibility is controlled using slacks is problematic (Bertsekas 1995) (see also the last comment in $\$ 3$ of Anitescu 2002a). In the specific setting of this paper, we are able to give the novel rule that guarantees convergence. The idea is to ensure that if $\left\{\beta^{k}\right\}$ were to be unbounded, then necessarily two things happen: $\left\|\mu^{k}\right\| \rightarrow+\infty$ and $d^{k} \rightarrow 0$. As will be established (see Proposition 5), this situation cannot occur, thus leading to boundedness of $\left\{\beta_{k}\right\}$.

The following simple lemma will be used several times in the sequel. 
Lemma 1. Let $\left\{x \mid g_{i}(x) \leq 0, i=1, \ldots, m\right\} \neq \varnothing$, where $g: \Re^{n} \rightarrow \Re^{m}$ is convex and continuously differentiable. Then for any $x \in \mathfrak{R}^{n}$ such that

$$
J(x):=\left\{i \mid g_{i}(x)>0\right\} \neq \varnothing,
$$

it holds that

$$
\sum_{i \in J(x)} \mu_{i} g_{i}^{\prime}(x)=0, \quad \mu_{i} \geq 0, i \in J(x) \Leftrightarrow \mu_{i}=0, i \in J(x) .
$$

Proof. By Gordan Theorem of the Alternatives (Mangasarian 1969), the assertion follows if we establish that

$$
\exists d \in \Re^{n} \quad \text { such that }\left\langle g_{i}^{\prime}(x), d\right\rangle<0, \quad i \in J(x) .
$$

We proceed to exhibit this $d$. Take any $y$ such that $g_{i}(y) \leq 0, i=1, \ldots, m$. By the convexity of $g$, we have that

$$
0>g_{i}(y)-g_{i}(x) \geq\left\langle g_{i}^{\prime}(x), y-x\right\rangle, \quad i \in J(x),
$$

thus establishing the claim.

We start by showing that the method is well defined. This is done in two steps: for the case where $d^{k}=0$ (but $t_{k}>0$ ), and when $d^{k} \neq 0$.

Proposition 2. Let $\left\{x \mid g_{i}(x) \leq 0, i=1, \ldots, m\right\} \neq \varnothing$, where $g: \Re^{n} \rightarrow \Re^{m}$ is convex and continuously differentiable.

If in Algorithm 1 we have $d^{k}=0$ and $t^{k}=0$, then $\left(x^{k}, \mu^{k}\right)$ is a KKT point of (1).

If $d^{k}=0$ and $t^{k}>0$, then there exists a finite integer $q$ such that Algorithm 1 generates $d^{k+q} \neq 0$.

Proof. Suppose that $d^{k}=0$ and $t^{k}=0$. Then (9) and (5) imply that $g_{i}\left(x^{k}\right) \leq 0$ for $i \in$ $I\left(x^{k}\right) \subset I_{k}$. Hence, $p\left(x^{k}\right)=0$. Using again (5), it is easy to see that $g_{i}\left(x^{k}\right)<0$ for all $i \notin I_{k}$. With this observation, setting $d^{k}=0$ and $t^{k}=0$ in (7)-(11), and further $\mu_{i}^{k}=0$ for $i \notin I_{k}$, we obtain KKT conditions for (1).

Suppose now that $d^{k}=0, t^{k}>0$, and $d^{k+q}=0, t^{k+q}>0$ for all integers $q$. Obviously, we then have $x^{k+q}=x^{k}$, by Step 4 of Algorithm 1. By (6), $p\left(x^{k+q}\right)=t^{k+q}(0)=t^{k+q}=p\left(x^{k}\right)=$ $t^{k}>0$. Then (11) implies that $\nu^{k+q}=0$. Using further (8), we have that

$$
\beta_{k+q}=\sum_{i \in I_{k+q}} \mu_{i}^{k+q} \text {. }
$$

In Step 5 of Algorithm 1, because $\left\|d^{k+q}\right\|^{-1}=+\infty$, (15) implies that $\beta_{k+q}<r_{k+q}$. Hence, by (14),

$$
\beta_{k+q+1}=\beta_{k+q}+\delta_{2} \text {. }
$$

This shows that $\beta_{k+q} \rightarrow+\infty$ as $q \rightarrow \infty$, and by (15),

$$
+\infty=\lim _{q \rightarrow \infty}\left\|\mu^{k+q}\right\| .
$$

Observe that because $t^{k+q}=p\left(x^{k+q}\right)$, (9) and (10) imply that $\mu_{i}^{k+q}=0$ for $i \in I_{k+q} \backslash I\left(x^{k+q}\right)$. Because $I\left(x^{k}\right)=I\left(x^{k+q}\right) \subset I_{k+q},(7)$ reduces to

$$
f^{\prime}\left(x^{k}\right)+\sum_{i \in I\left(x^{k}\right)} \mu_{i}^{k+q} g_{i}^{\prime}\left(x^{k}\right)=0 .
$$

Dividing both sides of the above equality by $\left\|\mu^{k+q}\right\|$ and passing onto the limit as $q \rightarrow \infty$, we conclude that

$$
\sum_{i \in I\left(x^{k}\right)} \tilde{\mu}_{i} g_{i}^{\prime}\left(x^{k}\right)=0, \quad \tilde{\mu}_{i} \geq 0, i \in I\left(x^{k}\right),\|\tilde{\mu}\|=1 .
$$

Because $p\left(x^{k}\right)>0$ implies that $\varnothing \neq I\left(x^{k}\right) \subset J\left(x^{k}\right)$, the latter contradicts Lemma 1, completing the proof. 
Solodov: Sequential Quadratically Constrained Quadratic Programming Methods Mathematics of Operations Research 29(1), pp. 64-79, () 2004 INFORMS

The following result shows that whenever $d^{k} \neq 0$, it is a descent direction for $\psi_{\beta_{k}}$ at $x^{k}$. This, in turn, implies that the linesearch step is well defined. Combining this fact with Proposition 2, it follows that the whole Algorithm 1 is well defined.

Proposition 3. Let $f, g_{i}: \Re^{n} \rightarrow \Re, i=1, \ldots, m$, be continuously differentiable. Then in Algorithm 1 it holds that

$$
\begin{aligned}
\psi_{\beta_{k}}^{\prime}\left(x^{k} ; d^{k}\right) & \leq \Delta_{k}-\frac{1}{2}\left\langle H^{k} d^{k}, d^{k}\right\rangle \\
& \leq-\left\langle H^{k} d^{k}, d^{k}\right\rangle-\frac{1}{2} \sum_{i \in I_{k}} \mu_{i}^{k}\left\langle G_{i}^{k} d^{k}, d^{k}\right\rangle-\nu^{k} p\left(x^{k}\right) .
\end{aligned}
$$

As a consequence, Step 3 of Algorithm 1 is well defined and terminates with some finite integer $j_{k}$.

Proof. As is well known and easy to see,

$$
\begin{aligned}
& \psi_{\beta_{k}}^{\prime}\left(x^{k} ; d^{k}\right) \\
& \quad=\left\langle f^{\prime}\left(x^{k}\right), d^{k}\right\rangle+\beta_{k}\left\{\begin{array}{ll}
0, & \text { if } I\left(x^{k}\right)=\varnothing \\
\max _{i \in I\left(x^{k}\right)}\left\langle g_{i}^{\prime}\left(x^{k}\right), d^{k}\right\rangle, & \text { if } p\left(x^{k}\right)>0 \\
\max _{i \in I\left(x^{k}\right)}\left[\left\langle g_{i}^{\prime}\left(x^{k}\right), d^{k}\right\rangle\right]_{+}, & \text {if } p\left(x^{k}\right)=0, I\left(x^{k}\right) \neq \varnothing
\end{array} .\right.
\end{aligned}
$$

Consider the three possible cases in (17):

If $I\left(x^{k}\right)=\varnothing$, then it must hold that $p\left(x^{k}\right)=0$ (and $g_{i}\left(x^{k}\right)<0$ for all $i$ ). Thus $0 \leq t^{k}=$ $t^{k}-p\left(x^{k}\right)$.

If $I\left(x^{k}\right) \neq \varnothing$, by 9 , for all $i \in I\left(x^{k}\right) \subset I_{k}$ it holds that

$$
\begin{aligned}
\left\langle g_{i}^{\prime}\left(x^{k}\right), d^{k}\right\rangle & \leq t^{k}-g_{i}\left(x^{k}\right)-\frac{1}{2}\left\langle G_{i}^{k} d^{k}, d^{k}\right\rangle \\
& \leq t^{k}-p\left(x^{k}\right),
\end{aligned}
$$

where the second inequality follows from $g_{i}\left(x^{k}\right)=p\left(x^{k}\right)$ for $i \in I\left(x^{k}\right)$, and $G_{i}^{k} \succeq O$.

If further $p\left(x^{k}\right)=0$, then $\left\langle g_{i}^{\prime}\left(x^{k}\right), d^{k}\right\rangle \leq t^{k}$. Then by the monotonicity of $[\cdot]_{+}$,

$$
\left[\left\langle g_{i}^{\prime}\left(x^{k}\right), d^{k}\right\rangle\right]_{+} \leq\left[t^{k}\right]_{+}=t^{k}-p\left(x^{k}\right) .
$$

Combining the three cases, (17) gives that

$$
\psi_{\beta_{k}}^{\prime}\left(x^{k} ; d^{k}\right) \leq\left\langle f^{\prime}\left(x^{k}\right), d^{k}\right\rangle+\beta_{k}\left(t^{k}-p\left(x^{k}\right)\right),
$$

which is the first inequality in the assertion (recalling the definition of $\Delta_{k}$ in (13)).

Multiplying both sides of (7) by $d^{k}$, we have that

$$
\left\langle f^{\prime}\left(x^{k}\right), d^{k}\right\rangle=-\left\langle H^{k} d^{k}, d^{k}\right\rangle-\sum_{i \in I_{k}} \mu_{i}^{k}\left(\left\langle g_{i}^{\prime}\left(x^{k}\right), d^{k}\right\rangle+\left\langle G_{i}^{k} d^{k}, d^{k}\right\rangle\right) .
$$

We further obtain

$$
\begin{aligned}
-\sum_{i \in I_{k}} \mu_{i}^{k}\left\langle g_{i}^{\prime}\left(x^{k}\right), d^{k}\right\rangle & =\sum_{i \in I_{k}} \mu_{i}^{k}\left(g_{i}\left(x^{k}\right)+\frac{1}{2}\left\langle G_{i}^{k} d^{k}, d^{k}\right\rangle-t^{k}\right) \\
& \leq\left(p\left(x^{k}\right)-t^{k}\right) \sum_{i \in I_{k}} \mu_{i}^{k}+\frac{1}{2} \sum_{i \in I_{k}} \mu_{i}^{k}\left\langle G_{i}^{k} d^{k}, d^{k}\right\rangle \\
& =\left(\beta_{k}-\nu^{k}\right)\left(p\left(x^{k}\right)-t^{k}\right)+\frac{1}{2} \sum_{i \in I_{k}} \mu_{i}^{k}\left\langle G_{i}^{k} d^{k}, d^{k}\right\rangle \\
& =\beta_{k}\left(p\left(x^{k}\right)-t^{k}\right)-\nu^{k} p\left(x^{k}\right)+\frac{1}{2} \sum_{i \in I_{k}} \mu_{i}^{k}\left\langle G_{i}^{k} d^{k}, d^{k}\right\rangle,
\end{aligned}
$$


where (10) was used in the first equality, (8) was used in the second equality, and (11) in the last. Combining the latter relation with (19) and (18), we obtain (16).

If $d^{k}=0$, Step 3 of Algorithm 1 returns $j_{k}=0$. If $d^{k} \neq 0$, for $\gamma \in[0,1]$ we have that

$$
\begin{aligned}
\psi_{\beta_{k}}\left(x^{k}+\gamma d^{k}\right) & =\psi_{\beta_{k}}\left(x^{k}\right)+\gamma \psi_{\beta_{k}}^{\prime}\left(x^{k} ; d^{k}\right)+o(\gamma) \\
& \leq \psi_{\beta_{k}}\left(x^{k}\right)+\gamma \Delta_{k}+o(\gamma),
\end{aligned}
$$

where the inequality is by (16). It follows that (12) is guaranteed to hold whenever $\gamma=\theta^{j}>0$ satisfies

$$
(1-\sigma) \gamma \Delta_{k} \leq o(\gamma)
$$

Because $\Delta_{k}<0$ when $d^{k} \neq 0$, the inequality above clearly holds for all $\gamma=\theta^{j}$ sufficiently small (i.e., all $j$ sufficiently large). Hence, Step 3 of Algorithm 1 terminates with some finite integer $j_{k}$.

We next establish that when close to the feasible region of (1), the solution of subproblem (4) is given by the solution of the subproblem without the slack variable:

$$
\begin{array}{ll}
\underset{d}{\operatorname{minimize}} & \left\langle f^{\prime}\left(x^{k}\right), d\right\rangle+\frac{1}{2}\left\langle H^{k} d, d\right\rangle, \\
\text { subject to } & g_{i}\left(x^{k}\right)+\left\langle g_{i}^{\prime}\left(x^{k}\right), d\right\rangle+\frac{1}{2}\left\langle G_{i}^{k} d, d\right\rangle \leq 0, \quad i \in A_{k},
\end{array}
$$

where $A_{k} \subset\{1, \ldots, m\}$. This fact will be used later to establish that the penalty parameters $\left\{\beta_{k}\right\}$ stay fixed from some point on, as well as for the local rate of convergence analysis. The part of Proposition 4 concerning boundedness of solutions and Lagrange multipliers of (20) is closely related to Fukushima et al. (2003, Lemma 3.3). However, we consider the case where possibly $G_{i}^{k} \neq g_{i}^{\prime \prime}\left(x^{k}\right)$ and $A_{k} \neq\{1, \ldots, m\}$.

Proposition 4. Let $f, g_{i}: \Re^{n} \rightarrow \mathfrak{R}, i=1, \ldots, m$, be continuously differentiable. Suppose that $g$ is convex and the Slater condition (2) is satisfied.

Let $\left\{x^{k}\right\}$ be any sequence converging to some $\bar{x} \in \Re^{n}$ such that $p(\bar{x})=0$, and let the matrices in (20) satisfy $\rho_{2} E \succeq H^{k} \succeq \rho_{1} E$ and $\rho_{2} E \succeq G_{i}^{k} \succeq O, i \in\{1, \ldots, m\}$, for all $k$, where $\rho_{2} \geq \rho_{1}>0$.

Then problem (20) is feasible (hence, solvable) for all $k$ sufficiently large and any $A_{k} \subset$ $\{1, \ldots, m\}$. Moreover, if $\left(s^{k}, \lambda^{k}\right)$ is a KKT point of $(20)$, then for any $\beta_{k} \geq\left\|\lambda^{k}\right\|_{1},\left(s^{k}, 0\right)$ is the unique solution of (4) with $I_{k}=A_{k}$. Conversely, if $\left(d^{k}, 0, \mu^{k}, \nu^{k}\right)$ is a KKT point of (4), then $\left(d^{k}, \mu^{k}\right)$ is a KKT point of (20).

Furthermore, the sequences $\left\{s^{k}\right\}$ and $\left\{\lambda^{k}\right\}$ are bounded.

Proof. Because $p(\bar{x})=0$, we have that $g_{i}(\bar{x})=0$ for $i \in I(\bar{x})$. By the convexity of $g$ and (2), it then holds that

$$
0>g_{i}(\hat{x})-g_{i}(\bar{x}) \geq\left\langle g_{i}^{\prime}(\bar{x}), \hat{x}-\bar{x}\right\rangle, \quad i \in I(\bar{x}) .
$$

Taking $d(\eta)=\eta(\hat{x}-\bar{x}), \eta \in(0,1]$, and using $\rho_{2} E \succeq G_{i}^{k}$, we have that

$$
\begin{gathered}
g_{i}\left(x^{k}\right)+\eta\left\langle g_{i}^{\prime}\left(x^{k}\right), \hat{x}-\bar{x}\right\rangle+\frac{\eta^{2}}{2}\left\langle G_{i}^{k}(\hat{x}-\bar{x}), \hat{x}-\bar{x}\right\rangle \\
\leq \eta\left(\left\langle g_{i}^{\prime}(\bar{x}), \hat{x}-\bar{x}\right\rangle+\frac{\eta \rho_{2}\|\hat{x}-\bar{x}\|^{2}}{2}\right)+\epsilon_{i}^{k},
\end{gathered}
$$

where, as $k \rightarrow \infty$,

$$
\epsilon_{i}^{k}=g_{i}\left(x^{k}\right)+\eta\left\langle g_{i}^{\prime}\left(x^{k}\right)-g_{i}^{\prime}(\bar{x}), \hat{x}-\bar{x}\right\rangle \rightarrow\left\{\begin{array}{ll}
g_{i}(\bar{x})=0, & i \in I(\bar{x}) \\
g_{i}(\bar{x})<0, & i \notin I(\bar{x})
\end{array} .\right.
$$


For each $i \in\{1, \ldots, m\}$ there exists $\eta_{i}>0$, and for $i \in I(\bar{x})$ also some $c_{i}>0$, such that

$$
\eta_{i}\left(\left\langle g_{i}^{\prime}(\bar{x}), \hat{x}-\bar{x}\right\rangle+\frac{\eta_{i} \rho_{2}\|\hat{x}-\bar{x}\|^{2}}{2}\right) \leq\left\{\begin{array}{ll}
-2 c_{i}, & i \in I(\bar{x}) \\
-g_{i}(\bar{x}) / 4, & i \notin I(\bar{x})
\end{array},\right.
$$

where (21) was used for $i \in I(\bar{x})$. Given those choices, for each $i \in\{1, \ldots, m\}$ there further exists an index $k_{i}$ such that

$$
\epsilon_{i}^{k} \leq\left\{\begin{array}{ll}
c_{i}, & i \in I(\bar{x}) \\
g_{i}(\bar{x}) / 2, & i \notin I(\bar{x})
\end{array}, \quad \forall k \geq k_{i} .\right.
$$

Denoting

$$
\bar{k}=\max _{i=1, \ldots, m} k_{i}, \quad \eta=\min _{i=1, \ldots, m} \eta_{i}, \quad c=\min \left\{\min _{i \in I(\bar{x})} c_{i},-\max _{i \notin I(\bar{x})} g_{i}(\bar{x}) / 4\right\},
$$

and using the two relations above and (22), we have that $d=\eta(\hat{x}-\bar{x})$ satisfies

$$
g_{i}\left(x^{k}\right)+\left\langle g_{i}^{\prime}\left(x^{k}\right), d\right\rangle+\frac{1}{2}\left\langle G_{i}^{k} d, d\right\rangle \leq-c<0 \quad \forall k \geq \bar{k}, i=1, \ldots, m .
$$

In particular, this shows that this $d$ is (strictly) feasible in (20) for any choice of $A_{k} \subset$ $\{1, \ldots, m\}$ and all $k \geq \bar{k}$.

Therefore, (20) is a feasible strongly convex program. Hence, it is (uniquely) solvable. Furthermore, by (24), it satisfies the Slater constraint qualification. Hence, there exist some $\lambda_{i}^{k} \in \Re, i \in A_{k}$, such that

$$
\begin{gathered}
f^{\prime}\left(x^{k}\right)+H^{k} s^{k}+\sum_{i \in A_{k}} \lambda_{i}^{k}\left(g_{i}^{\prime}\left(x^{k}\right)+G_{i}^{k} s^{k}\right)=0, \\
g_{i}\left(x^{k}\right)+\left\langle g_{i}^{\prime}\left(x^{k}\right), s^{k}\right\rangle+\frac{1}{2}\left\langle G_{i}^{k} s^{k}, s^{k}\right\rangle \leq 0, \quad \lambda_{i}^{k} \geq 0, i \in A_{k}, \\
\lambda_{i}^{k}\left(g_{i}\left(x^{k}\right)+\left\langle g_{i}^{\prime}\left(x^{k}\right), s^{k}\right\rangle+\frac{1}{2}\left\langle G_{i}^{k} s^{k}, s^{k}\right\rangle\right)=0, \quad i \in A_{k} .
\end{gathered}
$$

If $\beta_{k} \geq\left\|\lambda^{k}\right\|_{1}$, then Conditions (25)-(27) imply that $d^{k}=s^{k}, t^{k}=0, \nu^{k}=\beta_{k}-\left\|\lambda^{k}\right\|_{1}$ and $\mu_{i}^{k}=\lambda_{i}^{k}, i \in A_{k}=I_{k}$, satisfy KKT conditions (7)-(11). Hence, $\left(s^{k}, 0\right)$ is the solution of (4). Conversely, if $\left(d^{k}, 0, \mu^{k}, \nu^{k}\right)$ is a KKT point of (4), then $\left(d^{k}, \mu^{k}\right)$ is a KKT point of (20) for $A_{k}=I_{k}$, by direct inspection.

Next, note that because $d=\eta(\hat{x}-\bar{x})$ is feasible in (20) for all $k \geq \bar{k}$, we have that

$$
\begin{aligned}
\left\langle f^{\prime}\left(x^{k}\right), d\right\rangle+\frac{1}{2}\left\langle H^{k} d, d\right\rangle & \geq\left\langle f^{\prime}\left(x^{k}\right), s^{k}\right\rangle+\frac{1}{2}\left\langle H^{k} s^{k}, s^{k}\right\rangle \\
& \geq\left\|s^{k}\right\|\left(\frac{\rho_{1}\left\|s^{k}\right\|}{2}-\left\|f^{\prime}\left(x^{k}\right)\right\|\right),
\end{aligned}
$$

where the second inequality is by $H^{k} \succeq \rho_{1} E$. Because $\left\{f^{\prime}\left(x^{k}\right)\right\}$ is bounded and $\rho_{2} E \succeq H^{k}$, the above relation implies that $\left\{s^{k}\right\}$ must be bounded.

Suppose now that $\left\{\lambda^{k}\right\}$ is unbounded. We augment (25) by formally setting $\lambda_{i}^{k}=0$ for $i \in\{1, \ldots, m\} \backslash A_{k}$. Passing onto a subsequence, if necessary, we can assume that $\left\|\lambda^{k}\right\| \rightarrow \infty$ and $\left\{s^{k}\right\} \rightarrow \bar{s},\left\{H^{k}\right\} \rightarrow H,\left\{G_{i}^{k}\right\} \rightarrow G_{i}$. Dividing both sides of (25) by $\left\|\lambda^{k}\right\|$ and passing onto the limit as $k \rightarrow \infty$, we obtain that

$$
0=\sum_{i=1}^{m} \bar{\lambda}_{i}\left(g_{i}^{\prime}(\bar{x})+G_{i} \bar{s}\right), \quad \bar{\lambda} \geq 0,\|\bar{\lambda}\|=1 .
$$


By Gordan Theorem of the Alternatives (Mangasarian 1969), the latter is equivalent to

$$
\nexists s \in \Re^{n} \quad \text { such that }\left\langle g_{i}^{\prime}(\bar{x})+G_{i} \bar{s}, s\right\rangle<0 \quad \text { for } i \in\left\{i \mid \bar{\lambda}_{i}>0\right\} .
$$

Clearly, $\bar{\lambda}_{i}>0$ means that there exists an infinite subsequence of indices $\left\{k_{j}\right\}$ such that $i \in A_{k_{j}}$ and $\lambda_{i}^{k_{j}}>0$. For such $i$, (27) implies that

$$
g_{i}\left(x^{k_{j}}\right)+\left\langle g_{i}^{\prime}\left(x^{k_{j}}\right), s^{k_{j}}\right\rangle+\frac{1}{2}\left\langle G_{i}^{k_{j}} s^{k_{j}}, s^{k_{j}}\right\rangle=0 \quad \forall j .
$$

Passing onto the limit as $j \rightarrow \infty$, we obtain that

$$
g_{i}(\bar{x})+\left\langle g_{i}^{\prime}(\bar{x}), \bar{s}\right\rangle+\frac{1}{2}\left\langle G_{i} \bar{s}, \bar{s}\right\rangle=0, \quad i \in\left\{i \mid \bar{\lambda}_{i}>0\right\} .
$$

Passing onto the limit as $k \rightarrow \infty$ in (24), we also have that

$$
g_{i}(\bar{x})+\left\langle g_{i}^{\prime}(\bar{x}), d\right\rangle+\frac{1}{2}\left\langle G_{i} d, d\right\rangle \leq-c<0 .
$$

Substracting (29) from the latter inequality, we have that

$$
\begin{aligned}
0 & >\left\langle g_{i}^{\prime}(\bar{x}), d-\bar{s}\right\rangle+\frac{1}{2}\left\langle G_{i} d, d\right\rangle-\frac{1}{2}\left\langle G_{i} \bar{s}, \bar{s}\right\rangle \\
& =\left\langle g_{i}^{\prime}(\bar{x})+G_{i} \bar{s}, d-\bar{s}\right\rangle, \quad i \in\left\{i \mid \bar{\lambda}_{i}>0\right\},
\end{aligned}
$$

which contradicts (28). We conclude that $\left\{\lambda^{k}\right\}$ is bounded.

By (14), either $\beta_{k}$ is constant starting from some iteration index $k_{0}$ or it diverges to $+\infty$. We next show that the latter case cannot occur.

Proposition 5. Let $f, g_{i}: \Re^{n} \rightarrow \Re, i=1, \ldots, m$, be continuously differentiable. Suppose that $g$ is convex and that the Slater condition (2) is satisfied.

Let the sequence $\left\{x^{k}\right\}$ generated by Algorithm 1 be bounded, and $\rho_{2} E \succeq H^{k} \succeq \rho_{1} E$, $\rho_{2} E \succeq G_{i}^{k} \succeq O, i \in I_{k}$, for all $k$, where $\rho_{2} \geq \rho_{1}>0$.

Then there exists some iteration index $k_{0}$ such that

$$
\beta_{k}=\beta_{k_{0}} \quad \forall k \geq k_{0} .
$$

Proof. Suppose the opposite, i.e., $\beta_{k} \rightarrow+\infty$ as $k \rightarrow \infty$. Then (14) implies that $\beta_{k}<$ $r_{k}=\min \left\{\left\|d^{k}\right\|^{-1},\left\|\mu^{k}\right\|_{1}+\delta_{1}\right\}$ happens an infinite number of times. It then further follows that there exists a subsequence of iteration indices $\left\{k_{j}\right\}$, such that

$$
0=\lim _{j \rightarrow \infty} d^{k_{j}} \quad \text { and } \quad+\infty=\lim _{j \rightarrow \infty}\left\|\mu^{k_{j}}\right\| .
$$

Taking a further subsequence, if necessary, we can assume that $\left\{x^{k_{j}}\right\} \rightarrow \tilde{x}$ as $j \rightarrow \infty$. We next consider the two possible cases: $p(\tilde{x})>0$ and $p(\tilde{x})=0$.

Let $p(\tilde{x})>0$, and denote

$$
J(\tilde{x}):=\left\{i \mid g_{i}(\tilde{x})>0\right\} \neq \varnothing .
$$

Note that by the continuity of $g, J(\tilde{x}) \cap I_{k_{j}} \neq \varnothing$ for all $j$ large enough. By (9),

$$
g_{i}\left(x^{k_{j}}\right)+\left\langle g_{i}^{\prime}\left(x^{k_{j}}\right), d^{k_{j}}\right\rangle+\frac{1}{2}\left\langle G_{i}^{k_{j}} d^{k_{j}}, d^{k_{j}}\right\rangle \leq t^{k_{j}}, i \in I_{k_{j}} .
$$

For $i \in I_{k_{j}} \backslash J(\tilde{x})$, as $j \rightarrow \infty$ the left-hand side of the inequality above tends to $g_{i}(\tilde{x}) \leq 0$ (taking into account (30) and $\rho_{2} E \succeq G_{i}^{k} \succeq O$ ), while the right-hand side tends to $p(\tilde{x})>0$ (recall (6)). Hence, such constraints are inactive for all $j$ large enough, and by (10),

$$
\mu_{i}^{k_{j}}=0, \quad i \in I_{k_{j}} \backslash J(\tilde{x}) .
$$


Formally setting $\mu_{i}^{k_{j}}=0$ and $G_{i}^{k_{j}}=0$ for $i \in J(\tilde{x}) \backslash I_{k_{j}}$, we can write (7) as

$$
f^{\prime}\left(x^{k_{j}}\right)+H^{k_{j}} d^{k_{j}}+\sum_{i \in J(\tilde{x})} \mu_{i}^{k_{j}}\left(g_{i}^{\prime}\left(x^{k_{j}}\right)+G_{i}^{k_{j}} d^{k_{j}}\right)=0 .
$$

Dividing both sides of this equality by $\left\|\mu^{k_{j}}\right\|$ and passing onto the limit as $j \rightarrow \infty$, by (30) and $\rho_{2} E \succeq H^{k}, G_{i}^{k} \succeq O$, we obtain that

$$
0=\sum_{i \in J(\tilde{x})} \tilde{\mu} g_{i}^{\prime}(\tilde{x}), \quad \tilde{\mu}_{i} \geq 0, i \in J(\tilde{x}), \quad\left\|\tilde{\mu}_{i}\right\|=1,
$$

which contradicts Lemma 1.

Suppose now that $p(\tilde{x})=0$. By Proposition 4, subproblems (20) with $A_{k_{j}}=I_{k_{j}}$ are solvable for all indices $j$ large enough. Let $\left(s^{k_{j}}, \lambda^{k_{j}}\right)$ be a KKT point of (20). In particular, by Proposition $4,\left\{\lambda^{k_{j}}\right\}$ is bounded. Since $\beta_{k} \rightarrow+\infty$, we have that $\beta_{k_{j}}>\left\|\lambda^{k_{j}}\right\|_{1}$ and, again by Proposition 4, the unique solution of (4) is $d^{k_{j}}=s^{k_{j}}, t^{k_{j}}=0$, with multipliers $\left(\mu^{k_{j}}, \nu^{k_{j}}\right)$ generated by Algorithm 1. But then $\left(s^{k_{j}}, \mu^{k_{j}}\right)$ is a KKT point of (20), again by Proposition 4 . However, unboundedness of $\left\{\mu^{k_{j}}\right\}$ (see (30)) contradicts Proposition 4.

The proof is complete.

We are now ready to establish global convergence of Algorithm 1. Our results are stronger than Fukushima et al. (2003, Theorem 3.4) in some respects. First, we do not assume the convexity of $f$ or twice differentiability of $f$ and $g$. Second, we prove that the step sizes stay bounded away from zero, which is a desirable property not established in Fukushima et al. (2003). Additionally, even if the data are twice differentiable, we do not assume that $G_{i}^{k}=g_{i}^{\prime \prime}\left(x^{k}\right)$ and/or that the matrices $2 H^{k}-f^{\prime \prime}\left(x^{k}\right)+\sum_{i=1}^{m} \mu_{i}^{k} g_{i}^{\prime \prime}\left(x^{k}\right)$ are uniformly positive definite, as required in Fukushima et al. (2003, Theorem 3.4). Both Theorem 6 below and Fukushima et al. (2003, Theorem 3.4), assume the uniform boundedness and positive definiteness of $H^{k}$, and boundedness of the sequence $\left\{x^{k}\right\}$.

THEOREM 6. Let $f, g_{i}, i=1, \ldots, m$, be differentiable functions with Lipschitz-continuous derivatives. Let $g$ be convex, and the Slater condition (2) be satisfied.

Suppose further that in Algorithm 1 we have $\rho_{2} E \succeq H^{k} \succeq \rho_{1} E$ and $\rho_{2} E \succeq G_{i}^{k} \succeq O, i \in I_{k}$, for all $k$, where $\rho_{2} \geq \rho_{1}>0$, and that $I_{k}=\{1, \ldots, m\}$ for $k \geq k_{1}$.

If the sequence $\left\{x^{k}\right\}$ generated by Algorithm 1 is bounded, then the sequence $\left\{\mu^{k}\right\}$ is also bounded and every accumulation point of $\left\{\left(x^{k}, \mu^{k}\right)\right\}$ is a KKT point for problem (1). In particular, if $f$ is convex then every accumulation point of $\left\{x^{k}\right\}$ is a solution of (1).

Proof. We first show that the sequence of step sizes $\left\{\gamma_{k}\right\}$ is bounded away from zero. Let $L>0$ be the Lipschitz constant of $f^{\prime}, g_{i}^{\prime}, i=1, \ldots, m$. For all $\gamma \in[0,1]$ and any $i$, we have that

$$
\begin{aligned}
& {\left[g_{i}\left(x^{k}+\gamma d^{k}\right)\right]_{+}-\left[g_{i}\left(x^{k}\right)+\gamma\left\langle g_{i}^{\prime}\left(x^{k}\right), d^{k}\right\rangle\right]_{+}} \\
& \quad \leq\left[g_{i}\left(x^{k}+\gamma d^{k}\right)-g_{i}\left(x^{k}\right)-\gamma\left\langle g_{i}^{\prime}\left(x^{k}\right), d^{k}\right\rangle\right]_{+} \\
& \quad \leq\left|g_{i}\left(x^{k}+\gamma d^{k}\right)-g_{i}\left(x^{k}\right)-\gamma\left\langle g_{i}^{\prime}\left(x^{k}\right), d^{k}\right\rangle\right| \\
& \quad \leq \frac{L}{2} \gamma^{2}\left\|d^{k}\right\|^{2},
\end{aligned}
$$

where the first inequality follows from the fact that $[a-b]_{+} \geq[a]_{+}-[b]_{+} \forall a, b \in \mathfrak{R}$; and the last inequality is by the Lipschitz-continuity of $g_{i}^{\prime}$ (e.g., Bertsekas 1995, Proposition A.24). For $i \in I_{k}$, we further obtain

$$
\begin{aligned}
& {\left[g_{i}\left(x^{k}\right)+\gamma\left\langle g_{i}^{\prime}\left(x^{k}\right), d^{k}\right\rangle\right]_{+}} \\
& \quad=\left[\gamma\left(g_{i}\left(x^{k}\right)+\left\langle g_{i}^{\prime}\left(x^{k}\right), d^{k}\right\rangle\right)+(1-\gamma) g_{i}\left(x^{k}\right)\right]_{+}
\end{aligned}
$$




$$
\begin{aligned}
& \leq \gamma\left[g_{i}\left(x^{k}\right)+\left\langle g_{i}^{\prime}\left(x^{k}\right), d^{k}\right\rangle\right]_{+}+(1-\gamma)\left[g_{i}\left(x^{k}\right)\right]_{+} \\
& \leq \gamma\left[g_{i}\left(x^{k}\right)+\left\langle g_{i}^{\prime}\left(x^{k}\right), d^{k}\right\rangle+\frac{1}{2}\left\langle G_{i}^{k} d^{k}, d^{k}\right\rangle\right]_{+}+(1-\gamma)\left[g_{i}\left(x^{k}\right)\right]_{+} \\
& \leq \gamma t^{k}+(1-\gamma)\left[g_{i}\left(x^{k}\right)\right]_{+},
\end{aligned}
$$

where the second inequality is by the convexity of $[\cdot]_{+}$; and the last two are by the monotonicity of $[\cdot]_{+}$(taking also into account $G_{i}^{k} \succeq O$ and (9), (11)). Combining (31) and (32), we conclude that

$$
\left[g_{i}\left(x^{k}+\gamma d^{k}\right)\right]_{+} \leq \gamma t^{k}+(1-\gamma)\left[g_{i}\left(x^{k}\right)\right]_{+}+\frac{L}{2} \gamma^{2}\left\|d^{k}\right\|^{2}, \quad i \in I_{k} .
$$

By the Lipschitz-continuity of $f^{\prime}$, it also holds that

$$
f\left(x^{k}+\gamma d^{k}\right) \leq f\left(x^{k}\right)+\gamma\left\langle f^{\prime}\left(x^{k}\right), d^{k}\right\rangle+\frac{L}{2} \gamma^{2}\left\|d^{k}\right\|^{2} .
$$

For $k \geq k_{1}$, we then have that

$$
\begin{aligned}
\psi_{\beta_{k}}\left(x^{k}+\gamma d^{k}\right) & =f\left(x^{k}+\gamma d^{k}\right)+\beta_{k} p\left(x^{k}+\gamma d^{k}\right) \\
& \leq f\left(x^{k}\right)+\gamma\left\langle f^{\prime}\left(x^{k}\right), d^{k}\right\rangle+\beta_{k}(1-\gamma) p\left(x^{k}\right)+\gamma \beta_{k} t^{k}+\frac{L\left(1+\beta_{k}\right)}{2} \gamma^{2}\left\|d^{k}\right\|^{2} \\
& =\psi_{\beta_{k}}\left(x^{k}\right)+\gamma\left(\Delta_{k}-\frac{1}{2}\left\langle H^{k} d^{k}, d^{k}\right\rangle\right)+\frac{L\left(1+\beta_{k}\right)}{2} \gamma^{2}\left\|d^{k}\right\|^{2} \\
& \leq \psi_{\beta_{k}}\left(x^{k}\right)+\gamma \Delta_{k}+\frac{L\left(1+\beta_{k}\right)}{2} \gamma^{2}\left\|d^{k}\right\|^{2},
\end{aligned}
$$

where $I_{k}=\{1, \ldots, m\}$ was used in the first inequality, the second equality is by (13), and the last inequality is by $H^{k} \succeq O$.

By a direct comparison of the latter relation with (12), we conclude that (12) is guaranteed to be satisfied once $j$ is large enough, so that $\gamma=\theta^{j}$ falls within the set of $\gamma$, satisfying

$$
\frac{L\left(1+\beta_{k}\right)}{2} \gamma\left\|d^{k}\right\|^{2} \leq-(1-\sigma) \Delta_{k} .
$$

In particular, because Step 3 of Algorithm 1 did not accept the stepsize value $\theta^{k_{j}-1}>\theta^{k_{j}}$, it must be the case that

$$
k_{j}=0 \quad \text { or } \quad \gamma_{k} / \theta=\theta^{k_{j}-1}>\frac{-2(1-\sigma) \Delta_{k}}{L\left(1+\beta_{k}\right)\left\|d^{k}\right\|^{2}} .
$$

Hence,

$$
\gamma_{k} \geq \min \left\{1, \frac{-2 \theta(1-\sigma) \Delta_{k}}{L\left(1+\beta_{k}\right)\left\|d^{k}\right\|^{2}}\right\}, \quad k \geq k_{1} .
$$

By Proposition $5, \beta_{k}=\beta_{k_{0}}$ for all $k \geq k_{0}$ and some iteration index $k_{0}$. Furthermore, by (16) and $H^{k} \succeq \rho_{1} E$,

$$
-\Delta_{k} \geq \frac{1}{2}\left\langle H^{k} d^{k}, d^{k}\right\rangle \geq \rho_{1}\left\|d^{k}\right\|^{2} / 2
$$

We conclude that

$$
\gamma_{k} \geq \min \left\{1, \frac{(1-\sigma) \theta \rho_{1}}{L\left(1+\beta_{k_{0}}\right)}\right\}=\bar{\gamma}>0 \quad \forall k \geq k_{2}:=\max \left\{k_{0}, k_{1}\right\} .
$$


Now taking into account (12), (33), and (34), for $k \geq k_{2}$ we obtain that

$$
\begin{aligned}
\psi_{\beta_{k_{0}}}\left(x^{k}\right)-\psi_{\beta_{k_{0}}}\left(x^{k+1}\right) & \geq-\sigma \gamma_{k} \Delta_{k} \\
& \geq \sigma \bar{\gamma} \rho_{1}\left\|d^{k}\right\|^{2} / 2 .
\end{aligned}
$$

Because $\left\{x^{k}\right\}$ is bounded, $\left\{\psi_{\beta_{k_{0}}}\left(x^{k}\right)\right\}$ is also bounded, by continuity. By (35), it is also nonincreasing. Hence, it converges. Then (35) further implies that

$$
0=\lim _{k \rightarrow \infty} d^{k} .
$$

Furthermore, by Step 5 of Algorithm 1, the fact that $\beta_{k+1}=\beta_{k}=\beta_{k_{0}}$ implies that

$$
\beta_{k_{0}} \geq r_{k}=\min \left\{\left\|d^{k}\right\|^{-1},\left\|\mu^{k}\right\|_{1}+\delta_{1}\right\} .
$$

Taking into account (36), we conclude that there exists some $k_{3} \geq k_{0}$ such that

$$
\beta_{k_{0}}>\sum_{i \in I_{k}} \mu^{k} \quad \forall k \geq k_{3} .
$$

In particular, this means that $\left\{\mu^{k}\right\}$ is bounded and, using (8), that

$$
\nu^{k}>0 \quad \forall k \geq k_{3} .
$$

By (11), we then also have that

$$
t^{k}=0 \quad \forall k \geq k_{3} .
$$

Now passing onto the limit as $k \rightarrow \infty$ in (7)-(11) (where $I_{k}=\{1, \ldots, m\}$ ), we obtain that every accumulation point of $\left\{\left(x^{k}, \mu^{k}\right)\right\}$ satisfies the KKT conditions for problem (1). If $f$ is further convex, then every accumulation point of $\left\{x^{k}\right\}$ is a solution of (1).

As an immediate consequence of Theorem 6, we have the following fact.

COROLlaRY 7. Suppose that the assumptions of Theorem 6 are satisfied (except for the boundedness of $\left\{x^{k}\right\}$ ). If problem (1) has no KKT points, then the sequence $\left\{x^{k}\right\}$ generated by Algorithm 1 must be unbounded.

3. Local convergence. We next show that if the matrices $H^{k}$ and $G_{i}^{k}$ are (in a certain sense) asymptotically good approximations of $f^{\prime \prime}\left(x^{k}\right)$ and $g_{i}^{\prime \prime}\left(x^{k}\right)$, respectively, then in Algorithm 1 we have $\gamma_{k}=1$ for all $k$ sufficiently large. This implies that the Maratos effect does not occur. Then, under certain further assumptions, the quadratic rate of convergence is achieved.

Note that given the assumptions on $H^{k}$ needed for global convergence, the first condition in (38) implicitly subsumes that $f^{\prime \prime}\left(x^{k}\right)$ is positive definite for $k$ sufficiently large (unless some further analysis involving $d^{k}$ is performed). The latter is consistent with the assumptions of Theorem 6 below on the quadratic rate of convergence. In Algorithm 1, we can take the positive definite regularization $H^{k}=f^{\prime \prime}\left(x^{k}\right)+\rho_{k} E$, decreasing $\rho_{k} \geq 0$ appropriately as $x^{k}$ enters the region where $f^{\prime \prime}(x)$ is positive definite. This strategy is consistent with both the global and local convergence theory.

Approximation conditions (38) also resemble the quasi-Newton conditions of DennisMoré, but specific update rules are beyond the scope of this paper. Here, we can mention the situation where computing the second derivatives involves some numerical work (e.g., the approximation is done by finite differences). In that case, (38) imposes the natural requirement on the accuracy of such approximation. 
Proposition 8. Let the assumptions of Theorem 6 be satisfied. Suppose, in addition, that $f$ and $g$ are twice continuously differentiable, and

$$
\left\langle\left(f^{\prime \prime}\left(x^{k}\right)-H^{k}\right) d^{k}, d^{k}\right\rangle=o\left(\left\|d^{k}\right\|^{2}\right), \quad\left\langle\left(g_{i}^{\prime \prime}\left(x^{k}\right)-G_{i}^{k}\right) d^{k}, d^{k}\right\rangle=o\left(\left\|d^{k}\right\|^{2}\right), i \in I_{k} .
$$

Then there exists an iteration index $k_{4}$ such that

$$
\gamma_{k}=1 \quad \forall k \geq k_{4} .
$$

Proof. By Theorem $6, \beta_{k}=\beta_{k_{0}}, t^{k}=0$ for $k \geq k_{3}$ (where $k_{3}$ is defined above), and $d^{k} \rightarrow 0$.

For $i \in I_{k}$ and $k \geq k_{3}$, we have that

$$
\begin{aligned}
{\left[g_{i}\left(x^{k}+d^{k}\right)\right]_{+}=} & {\left[g_{i}\left(x^{k}+d^{k}\right)\right]_{+}-\left[g_{i}\left(x^{k}\right)+\left\langle g_{i}^{\prime}\left(x^{k}\right), d^{k}\right\rangle+\frac{1}{2}\left\langle G_{i}^{k} d^{k}, d^{k}\right\rangle\right]_{+} } \\
\leq & \left|g_{i}\left(x^{k}+d^{k}\right)-g_{i}\left(x^{k}\right)-\left\langle g_{i}^{\prime}\left(x^{k}\right), d^{k}\right\rangle-\frac{1}{2}\left\langle g_{i}^{\prime \prime}\left(x^{k}\right) d^{k}, d^{k}\right\rangle\right| \\
& +\frac{1}{2}\left|\left\langle\left(g_{i}^{\prime \prime}\left(x^{k}\right)-G_{i}^{k}\right) d^{k}, d^{k}\right\rangle\right| \\
= & o\left(\left\|d^{k}\right\|^{2}\right),
\end{aligned}
$$

where the first equality follows from (9), where $t^{k}=0$, and the inequality follows from the fact that $[a]_{+}-[b]_{+} \leq|a-b|$ for any $a, b \in \Re$. Because $I_{k}=\{1, \ldots, m\}$ for $k \geq k_{3}$, we further obtain that

$$
\begin{aligned}
\psi_{\beta_{k_{0}}}\left(x^{k}+d^{k}\right) & =f\left(x^{k}+d^{k}\right)+\beta_{k_{0}} p\left(x^{k}+d^{k}\right) \\
& =f\left(x^{k}+d^{k}\right)+o\left(\left\|d^{k}\right\|^{2}\right) \\
& =f\left(x^{k}\right)+\left\langle f^{\prime}\left(x^{k}\right), d^{k}\right\rangle+\frac{1}{2}\left\langle f^{\prime \prime}\left(x^{k}\right) d^{k}, d^{k}\right\rangle+o\left(\left\|d^{k}\right\|^{2}\right) \\
& =\psi_{\beta_{k_{0}}}\left(x^{k}\right)-\beta_{k_{0}} p\left(x^{k}\right)+\left\langle f^{\prime}\left(x^{k}\right), d^{k}\right\rangle+\frac{1}{2}\left\langle H^{k} d^{k}, d^{k}\right\rangle+o\left(\left\|d^{k}\right\|^{2}\right) \\
& =\psi_{\beta_{k_{0}}}\left(x^{k}\right)+\Delta_{k}+o\left(\left\|d^{k}\right\|^{2}\right),
\end{aligned}
$$

where the second equality is by (39), the fourth equality is by (38), and the last is by the definition of $\Delta_{k}$ given in (13) and $t^{k}=0$.

In view of the last relation and (12), to establish that $\gamma_{k}=1$ in Algorithm 1, it is enough to show that

$$
(1-\sigma) \Delta_{k}+o\left(\left\|d^{k}\right\|^{2}\right) \leq 0
$$

for all $k$ sufficiently large. By (16) and $H^{k} \succeq \rho_{1} E$, we have that

$$
\Delta_{k} \leq-\frac{1}{2}\left\langle H^{k} d^{k}, d^{k}\right\rangle \leq-\frac{\rho_{1}\left\|d^{k}\right\|^{2}}{2},
$$

which verifies (40).

REMARK 9. If we assume that the method converges, then some of the assumptions in Proposition 8 can be relaxed. First, one no longer has to ask that $I_{k}=\{1, \ldots, m\}$ for $k$ large enough. This is easy to see, because $d^{k} \rightarrow 0$, and for $i \notin I\left(x^{k}\right)$ we then have $g_{i}\left(x^{k}\right)+\left\langle g_{i}^{\prime}\left(x^{k}\right), d^{k}\right\rangle+\frac{1}{2}\left\langle G_{i}^{k} d^{k}, d^{k}\right\rangle<0$ for $k$ large enough. Hence, the first equality leading to (39) is then valid also for $i \in\{1, \ldots, m\} \backslash I_{k}$. Then the rest of the proof applies. 
Additionally, instead of $H^{k} \succeq \rho_{1} E$, we could assume that $H^{k}+\sum_{i \in I_{k}} \mu_{i}^{k} G_{i}^{k} \succeq \rho_{1} E$. Indeed, (16) implies

$$
\Delta_{k} \leq-\frac{1}{2}\left\langle\left(H^{k}+\sum_{i \in I_{k}} \mu_{i}^{k} G_{i}^{k}\right) d^{k}, d^{k}\right\rangle
$$

from which then (40) also follows.

Theorem 6 (recall in particular (37)) and Proposition 4 imply that Algorithm 1 eventually reduces to SQCQP method based on subproblems (20) without the slack variable. Furthermore, by Proposition 8, the unit step size is always accepted from some point on. The local quadratic rate of convergence now follows essentially from Fukushima et al. (2003, Theorem 4.7).

THEOREM 10. Let $f, g_{i}, i=1, \ldots, m$, be convex twice continuously differentiable functions with Lipschitz-continuous second derivatives, and suppose that the Slater condition (2) holds. Let $\bar{x} \in \Re^{n}$ be the (unique) solution of (1) such that $f^{\prime \prime}(\bar{x})+\sum_{i=1}^{m} \bar{\mu} g_{i}^{\prime \prime}(\bar{x})$ is positive definite for any $\bar{\mu} \in M$, where $M \subset \Re^{m}$ is the set of Lagrange multipliers assiciated with $\bar{x}$.

Then there exists a neighbourhood $X$ of $\bar{x}$ such that the sequence $\left\{x^{k}\right\}$ generated by Algorithm 1 with $x^{0} \in X$ and $H^{k}=f^{\prime \prime}\left(x^{k}\right), G_{i}^{k}=g_{i}^{\prime \prime}\left(x^{k}\right)$ for all $k$, converges to $\bar{x}$ at a quadratic rate. Furthermore, $\operatorname{dist}\left(\mu^{k}, M\right) \rightarrow 0$ at a quadratic rate.

4. Concluding remarks. We presented a sequential quadratically constrained quadratic programming algorithm with a number of desirable convergence and implementation features. Specifically:

- The feasibility of subproblems is automatic.

- Convexity of the objective function is not needed for global convergence.

- Twice differentiability of the problem data is not needed for global convergence.

- The step size in the linesearch procedure is always bounded away from zero.

-If the problem data are twice continuously differentiable and the method uses asymptotically good approximations of second-order derivatives, then the unit step size is eventually always accepted.

- Thus the Maratos effect does not occur, and quadratic convergence is achieved under certain assumptions.

Acknowledgments. The author thanks the two anonymous referees for their comments, which led to an improvement of the original version of this paper. This research was partially supported by CNPq Grant 300734/95-6, PRONEX-Optimization, and FAPERJ.

\section{References}

Anitescu. M. 2002a. On the rate of convergence of sequential quadratic programming with nondifferentiable exact penalty function in the presence of constraint degeneracy. Math. Programming 92 359-386.

Anitescu, M. 2002b. A superlinearly convergent sequential quadratically constrained quadratic programming algorithm for degenerate nonlinear programming. SIAM J. Optim. 12 949-978.

Bertsekas, D. P. 1995. Nonlinear Programming. Athena Scientific, Belmont, MA.

Boggs, B. T., J. W. Tolle. 1996. Sequential quadratic programming. Acta Numerica 4 1-51.

Bonnans, J. F. 1989. Asymptotic admissibility of the unit stepsize in exact penalty methods. SIAM J. Control Optim. 27 631-641.

Bonnans, J. F., J. Ch. Gilbert, C. Lemaréchal, C. Sagastizábal. 2003. Numerical Optimization: Theoretical and Practical Aspects. Springer-Verlag, Berlin, Germany.

Fukushima, M. 1986. A successive quadratic programming algorithm with global and superlinear convergence properties. Math. Programming 35 253-264.

Fukushima, M., Z-Q. Luo, P. Tseng. 2003. A sequential quadratically constrained quadratic programming method for differentiable convex minimization. SIAM J. Optim. 13 1098-1119.

Kruk, S., H. Wolkowicz. 2000. Sequential, quadratically constrained, quadratic programming for general nonlinear programming. H. Wolkowicz, R. Saigal, L. Vandenberghe, eds. Handbook of Semidefinite Programming. Kluwer Academic Publishers, Boston, MA, 563-575. 
Solodov: Sequential Quadratically Constrained Quadratic Programming Methods

Lobo, M. S., L. Vandenberghe, S. Boyd, H. Lebret. 1998. Applications of second-order cone programming. Linear Algebra and Its Appl. 284 193-228.

Mangasarian, O. L. 1969. Nonlinear Programming. McGraw-Hill, New York.

Maratos, N. 1978. Exact penalty function algorithms for finite dimensional and control optimization problems. Ph.D. thesis, Imperial College, University of London, London, U.K.

Mayne, D. Q., E. Polak. 1982. A superlinearly convergent algorithm for constrained optimization problems. Math. Programming Stud. 16 45-61.

Monteiro, R. D. C., T. Tsuchiya. 2000. Polynomial convergence of primal-dual algorithms for the second-order cone programs based on the MZ-family of directions. Math. Programming 88 61-83.

Nesterov, Y. E., A. S. Nemirovskii. 1993. Interior Point Polynomial Methods in Convex Programming: Theory and Applications. SIAM Publications, Philadelphia, PA.

Panin, V. M. 1979. A second-order method for discrete min-max problem. USSR Comput. Math. and Math. Phys. 19 90-100.

Panin, V. M. 1981. Some methods of solving convex programming problems. USSR Comput. Math. and Math. Phys. 21 57-72.

Powell, M. J. D. 1983. Variable metric methods for constrained optimization. A. Bachem, M. Grötschel, B. Korte, eds. Mathematical Programming: The State of Art. Springer-Verlag, Berlin, Germany, 288-311.

Tsuchiya, T. 1999. A convergence analysis of the scale-invariant primal-dual path-following algorithms for secondorder cone programming. Optim. Methods Software 11 141-182.

Wiest, E. J., E. Polak. 1992. A generalized quadratic programming-based phase-I-phase-II method for inequalityconstrained optimization. Appl. Math. Optim. 26 223-252. 